\title{
Relaciones familiares versus aprendizaje: un análisis con niños de 5 y 6 años
}

\section{Family relationships versus learning: an analysis with children aged 5 and 6 years}

Investigación

\author{
Marisa Claudia Jacometo Durante \\ Faculdade la Salle-Brasil \\ marisa@faculdadelasalle.edu.br \\ Adriana Rossato Yanagu \\ Faculdade la Salle-Brasil \\ adriana12@gmail.com
}

Recibido: 02 de diciembre de 2016 / Aceptado: 19 de enero de 2017

\section{Resumen}

Como la primera mediadora entre el hombre y la cultura, la familia constituye la unidad dinámica de las relaciones de naturaleza afectiva, social y cognitiva que están inmersas en las condiciones materiales, históricas y culturales de un grupo social determinado. El objetivo general del estudio fue investigar si las relaciones familiares pueden comprometer el aprendizaje de los niños. Específicamente, se pretende: a) Identificar los conflictos en las relaciones familiares; b) Verificar si los conflictos en las relaciones familiares pueden comprometer las relaciones interpersonales de los niños en fase de alfabetización; c) Analizar si el conflicto en las relaciones familiares afecta el aprendizaje de los niños. En el estudio participaron 219 familias con niños de 5 y 6 años. Los principales resultados indican que existe un distanciamiento de los padres en relación a la vida escolar de los hijos, así como en las relaciones familiares ocurre una mezcla de conflicto y afecto. Se considera que el aprendizaje de los niños puede verse comprometido por los conflictos en las relaciones familiares en virtud de que el aprendizaje y el desempeño escolar dependen, en primer lugar, de la interacción familiar y posteriormente de la relación entre profesor y alumno.

Descriptores: Relaciones familiares, aprendizaje, niños, afecto, conflicto.

\begin{abstract}
As the first mediator between man and culture, the family constitutes the dynamic unity of affective, social and cognitive relations that are immersed in the material, historical and cultural conditions of a given social group. The overall objective of the study was to investigate whether family relationships can compromise children's learning. Specifically, it was intended to: a) Identify conflicts in family relationships; B) To verify if the conflicts in the family relations can compromise the interpersonal relationship of the children in the literacy phase; C) Analyze whether conflicts in family relationships interfere with children's learning. A total of 219 families with children aged 5 and 6 participated in the study. The main results indicate that there is a distancing of the parents in relation to the school life of the children, as well as in the family relationships there is a mixture of conflict and affection. It is considered that the learning of children can be compromised by conflicts in family relationships because learning and school performance depend primarily on family interaction and later on the relationship between teacher-student. Keywords: Family relationships, learning, children, affectivity, conflicts.
\end{abstract}

Forma sugerida de citar: Jacometo Durante, Marisa \& Rossato Yanagu, Adriana (2017). Relaciones familiares versus aprendizaje: un análisis con niños de 5 y 6 años. Alteridad, 12(1), pp. 55-66. 


\section{Introducción}

La familia como una unidad dinámica, a lo largo de la historia ha sufrido cambios constantes, por lo que es difícil seguir un solo curso. Ribeiro (1999) entiende su concepto como una tarea compleja, principalmente cuando se toma como principal referencia sus valores, sus creencias y experiencias personales. La tendencia, según la autora, es conceptualizar la familia a partir de nuestras propias familias. De esta manera, se vuelve fundamental despojarse de los valores, creencias y experiencias familiares personales. Como señala Mioto (1997), cuando hablamos de "familias" debemos atenernos a su especificidad y su particularidad, puesto que las familias difieren significativamente entre sí en los diversos momentos de la historia humana.

En términos jurídicos, Genofre (1997) explica que la familia, originalmente en el derecho romano, era patriarcal y considerada como todo lo que estuviese bajo el poder paterno: esposa, hijos, esclavos e incluso bienes, como tierras, herramientas y animales de trabajo. La tradición romana se constituye en un fuerte factor de influencia en las legislaciones modernas. Bajo la ley brasileña, en que prevalecen los derechos canónico y portugués, en los cuales la Iglesia representaba el concepto principal de familia. El derecho civil, bajo la influencia de la Iglesia, mantuvo durante mucho tiempo el principio de la indisolubilidad del matrimonio.

Con la Constitución Federal (1988), el matrimonio dejó de ser la piedra angular de la familia. Independientemente de la existencia del matrimonio civil o religioso, se considera como una unidad familiar la unión estable entre un hombre y una mujer. También se considera como una entidad familiar a la comunidad formada por uno de los padres y sus descendientes, encajándose en la definición de familias monoparentales, en las que el hombre o la mujer asume las responsabilidades.

En Brasil, el Código de Derecho Civil (2015) asegura la protección a las familias porque se consideran la base de la sociedad y las conceptúa como un conjunto de personas unidas por un vínculo jurídico de carácter familiar. La constitución de la familia aún se forma a través del matrimonio entre el hombre y la mujer, estableciendo una unión plena de vida, sobre la base de la igualdad de derechos y deberes de los cónyuges.

Las familias se constituyen, de modo general, fundamentadas en sentimientos de amor y solidaridad, con el propósito de preservar los lazos afectivos de atención, afecto, cuidado y protección de la pareja y los hijos, en busca de la realización de proyectos de manera compartida.

En el contexto de la afectividad, se encuentra el proceso de aprendizaje y es en la base de la familia donde se desarrollan los primeros hábitos, que garantizan o no, una base sólida y segura para enfrentar las adversidades.

Por lo tanto, la relevancia del estudio está en dilucidar que los conflictos en las relaciones familiares influyen en la formación de las relaciones interpersonales de los hijos, así como en el proceso de aprendizaje.

Esta investigación se llevó a cabo a través de un estudio de caso, que de acuerdo con Yin (2016), es más que un método y constituye una estrategia de investigación, al ser el estudio profundo y sistemático de un grupo, una situación o persona que sea representativa en su universo de investigación. En este sentido, se estudió un grupo de familias con niños de entre 5 y 6 años que están en etapa de educación infantil. El método de enfoque es inductivo para permitir, de acuerdo al método, que los datos conduzcan a la aparición del concepto, de acuerdo a (Yin, 2016). El análisis fue cualitativo debido a la comprensión de los significados situacionales presentados por los participantes (Richardson, 2010). También se caracteriza por ser descriptivo, como se describe en los fenómenos encontrados en las 219 familias encuestadas, mediante el uso de un cuestionario mixto (Richardson, 2010).

Para la revisión bibliográfica se llevaron a cabo lecturas en libros y revistas especializados en el área de educación, así como consultas en artículos publicados. 
El cuestionario se organizó con el fin de responder a los objetivos específicos. Se utilizaron preguntas objetivas y argumentativas (mixto) que permitan alcanzar los objetivos propuestos por la investigación.

El cuestionario fue aplicado a 219 familias con niños de 5 y 6 años de edad residentes en diferentes barrios de la ciudad de Lucas de Río Verde. Estos niños estudian en escuelas públicas y privadas. La muestra fue intencional, pues se delimitó al rango de edad y el criterio de selección fue la aceptación por parte de la familia para participar en la investigación.

La tabulación de los datos fue organizada de manera que las alternativas fueran tabuladas $\mathrm{y}$ las disertativas agrupadas por similitudes $\mathrm{y}$ diferencias.

Para el análisis de datos buscados se buscó coherencia con la revisión de la literatura, utilizando el método inductivo, es decir, la interpretación.

\section{Revisión de la literatura}

\section{El contexto familiar}

La familia, presente en todas las sociedades, es uno de los primeros entornos de socialización de los individuos, que actúa como mediador principal de los patrones, modelos e influencias culturales (Duarte, 1994). También se la considera la primera institución social que, junto con otras, busca asegurar la continuidad y el bienestar de sus miembros y de la comunidad, incluyendo el bienestar y la protección del niño. Por lo tanto, tiene un impacto significativo y una fuerte influencia en el comportamiento de los individuos, especialmente los niños, que aprenden las diferentes maneras de existir, de ver el mundo y de construir sus relaciones sociales.

Como primera mediadora entre el hombre y la cultura, la familia constituye la unidad dinámica de las relaciones de naturaleza afectiva, social y cognitiva que están inmersas en las condiciones materiales, históricas y culturales de un grupo social determinado. Ella es la matriz del aprendizaje humano, con significados y prácticas culturales propias que generan modelos de relaciones interpersonales y de construcción individual y colectiva. Los acontecimientos y experiencias familiares proporcionan la formación de repertorio conductual, de acciones y resoluciones de problemas con significados universales y particulares.

Estas experiencias integran la experiencia colectiva e individual que organiza, interfiere y la vuelve una unidad dinámica, estructurando las formas de subjetividad e interacción social. Y es a través de las interacciones familiares que se concretan las transformaciones en las sociedades que, a su vez, influirán en las relaciones familiares futuras, que se caracterizan por un proceso de influencias bidireccionales entre los miembros de la familia y los diferentes ambientes que componen los sistemas sociales, entre ellos la escuela, que constituyen un factor importante para el desarrollo de la persona (Duarte, 1994). Por lo tanto, ella es la principal responsable de la incorporación de los cambios sociales e intergeneracionales ocurridos a lo largo del tiempo, con los padres ejerciendo un papel preponderante en la construcción de la persona, su personalidad y su inclusión en el mundo laboral y social (Mioto, 1997).

En el entorno familiar los niños aprenden a gestionar y resolver conflictos, a controlar las emociones, a expresar los diferentes sentimientos que constituyen las relaciones interpersonales, a lidiar con las diversidades y las adversidades de la vida. Estas habilidades sociales y su forma de expresión, desarrolladas inicialmente en el ámbito familiar, tienen repercusiones en otros entornos con los que el niño, adolescente o adulto interactúan, provocando aspectos saludables o causando problemas y alterando la capacidad de aprendizaje (Mioto, 1997).

\section{Estudios sobre el afecto}

Este tema aborda la perspectiva sobre la afectividad, con estudios de Piaget, Wallon y otros autores contemporáneos. 
La tesis de Piaget (1977) sobre las relaciones entre afectividad e inteligencia, es que ambas son indisociables e integradas al desarrollo psicológico, no es posible que se trate de dos psicologías, una de la afectividad y otra de la inteligencia para explicar los comportamientos. Se sostiene que la afectividad no se limita solamente a las emociones y sentimientos, sino que también incluye la forma en que se cumplan las tendencias y la voluntad del niño.

Por afectividad se entiende los sentimientos propiamente dichos y, en particular, las emociones; las diversas tendencias, incluyendo las "tendencias superiores" y, en particular la voluntad (Cavenaghi y Saltini, 2014, p. 39).

Según Piaget (1977, p. 265), es indiscutible que el afecto juega un papel esencial en el funcionamiento de la inteligencia. Sin amor no habría ningún interés, necesidad o motivación. El afecto es una condición necesaria en la constitución de la inteligencia. Para él, "el afecto y la cognición resultan de una adaptación continua e interdependiente, en la que los sentimientos expresan los intereses y los valores de las acciones y de las estructuras inteligentes". "Si bien los esquemas afectivos conducen a la formación del carácter, los esquemas cognitivos conducen a la construcción de la inteligencia".

Las experiencias familiares fomentan la formación del repertorio de comportamientos, de acciones y resoluciones frente a los problemas (Dessen, Polonia, 2007). Es en el ambiente familiar donde también el niño aprende a hacer frente a los conflictos, a controlar sus emociones, a demostrar los diferentes sentimientos que impregnan las relaciones y a lidiar con la vida $y$ sus dificultades.

Para comprender el papel de las emociones en la teoría psicogénica de Piaget (1977), es necesario considerar y reflexionar sobre la asociación que él establece entre la génesis de la moral y la formación de la personalidad. En su obra, él presenta la cuestión de la génesis de la moral relacionada con la construcción de la escala de valores, las ideas y los sentimientos morales.
Para el teórico, toda moral está formada por un sistema de reglas y la moralidad consiste en el respeto que el individuo tiene de esas reglas. Los niños que están en el período pre-operatorio se encuentran todavía en la etapa de la heteronomía moral donde las reglas son externas, sagradas e inmutables, impuestas por los adultos. Se entiende entonces, la seriedad con la que debe llevarse a cabo el proceso de formación de estos individuos.

En este sentido, el psicólogo León Festinger (1957) propuso una teoría de disonancia cognitiva centrada en cómo las personas tratan de lograr la coherencia interna. Él sugirió que las personas tienen una necesidad interior para asegurarse de que sus creencias y comportamientos son consistentes. Creencias inconsistentes o conflictivas conducen a la discordia, que las personas se esfuerzan en evitar.

El afecto se refiere al conjunto de emociones positivas que existen en las relaciones interpersonales (Benetti, 2006). Eso implica una relación de cariño y cuidado que se tiene con alguien. Los lazos afectivos proporcionan apoyo psicológico y social en la familia, ayudando a hacer frente a los problemas cotidianos; el conflicto, sin embargo, se caracteriza por sentimientos negativos que pueden llevar al estrés y la agresividad en el sistema familiar.

Según Piaget (1977) el proceso de desarrollo pasa por la dimensión social, e implica la cognición, afecto y moral. El elemento de gran alcance que es la afectividad influye en nuestras actividades intelectuales y a esta selección de actividades, Piaget (1977) las llama de interés, que no son desarrolladas por lo cognitivo, sino por lo afectivo.

En este sentido, Cavenaghi; Saltini (2014) sugieren que el gran desafío de la educación sería favorecer el desarrollo intelectual en armonía con el desarrollo afectivo moral, de modo que el sujeto pueda alcanzar su autonomía intelectual, afectiva y moral, basado en leyes de reciprocidad construidas en sus interacciones con el entorno físico, social, histórico y cultural. El maestro no debe estar comprometido apenas con la construcción del conocimiento del estudiante, sino un profesio- 
nal involucrado con el desarrollo de la autonomía cognitiva, moral, social y emocional.

Por lo tanto, el enfoque de Henri Wallon privilegia el aspecto afectivo, lo que indica su papel fundamental en el inicio de la vida del niño. Para este autor (1995), la emoción organiza la vida psíquica inicial y precede a las primeras construcciones cognitivas. Define el desarrollo como el paso del yo orgánico al yo psíquico a través de las primeras emociones que son, en esencia, el instrumento para interactuar con el otro, antes de que se construya la cognición. Wallon (1995) presenta lo que él considera como la secuencia del desarrollo en relación a la construcción de la persona a partir de la idea del papel estructurador de las primeras emociones en relación con la cognición, para después describir el desarrollo como un movimiento de alternancia de predominancias, a veces emocional, a veces cognitiva culminando con una preponderancia cognitiva.

Las medidas propuestas por Wallon son: 1) falta de diferenciación entre yo y el otro (en la cual las primeras emociones hacen la comunicación y la expresión de las necesidades orgánicas); 2) diferenciación progresiva entre yo y el otro con el despuntar de la persona (inicialmente más para oponerse al mundo en la fase de oposición, para después afirmar su yo en la fase de personalismo); 3) llegando (aproximadamente en la edad escolar) a la etapa categorial, en la cual, ya están en posesión los instrumentos cognitivos tales como la representación y el pensamiento racional, utilizados para coordinar las emociones y construir conocimientos.

Wallon (1995) afirma que la afectividad constituye un papel fundamental en la formación de la inteligencia, con el fin de determinar los intereses y necesidades individuales de la persona. Se atribuye a las emociones un papel clave en la formación de la vida psíquica, un vínculo entre lo social y lo orgánico. Así se puede entender que, bajo la influencia del medio, las emociones tienden a realizar, a través de manifestaciones intensas, una conexión entre el individuo y el aprendizaje.
Va en contra de la naturaleza tratar al niño de manera fragmentaria. En cada edad, ella constituye un conjunto indisociable y original. En la sucesión de sus edades, ella es un ser único en curso de metamorfosis. Hecha de contrastes y conflictos, su unidad será, por eso aún más susceptible al desarrollo y la innovación (Wallon, 1995, p.198).

\section{Aprendizaje}

Vygotsky (1993) expone la idea de que todos los seres humanos son capaces de aprender, pero es necesario que se adapte a nuestra forma de enseñar. Vale la pena recordar que el aprendizaje implica separarse, crecer, tomar conciencia de la diferencia, entendiendo que esta conciencia humaniza y abre camino a lo subjetivo.

Piaget (1977) afirma que el aprendizaje es necesariamente un proceso de equilibrio, ya que hace que el sistema cognitivo busque nuevas maneras de interpretar y comprender la realidad, dado que se aprende educando.

Según Piaget (1977), el niño es idealizado como un ser activo que en todo momento interactúa con la realidad, actuando activamente con objetos y personas. Esa interacción con el medio hace que construya estructuras mentales $\mathrm{y}$ adquiera maneras de hacer que funcionen. El punto central, por lo tanto, es el intercambio entre el organismo y el medio, y esa correlación se produce a través de dos procesos simultáneos: la organización interna y la adaptación al medio, funciones ejercidas por el organismo durante toda la vida. La adecuación definida por Piaget (1977), como el propio desarrollo de la inteligencia, se llevan a cabo a través de la asimilación y la acomodación. Los esquemas de asimilación se transforman y se configuran conforme avanzan las fases de desarrollo.

Se considera todavía que el proceso de desarrollo está influenciado por factores tales como la maduración (crecimiento biológico de órganos), la ejercitación (funcionamiento de los sistemas y órganos que implican la formación 
de hábitos), el aprendizaje social (adquisición de valores, lenguaje, costumbres y las normas culturales y sociales) y equilibrio (proceso de autorregulación interna del organismo, que se constituye en la búsqueda sucesiva de reequilibrio después de cada desequilibrio sufrido).

Según Piaget (1977), el período preoperatorio comienza a los 02 años y se extiende hasta los 07 años, caracterizado por la aparición de la función simbólica o semiótica, es decir, la manifestación de lenguaje que marca el paso del período sensorio-motor para el preoperatorio.

El niño pre-operacional ve las cosas desde su punto de vista. Él piensa que todas las personas piensan como él y, como considera sus pensamientos correctos, nunca los cuestiona. Asimila todo a sí mismo y a su punto de vista, a servicio de sus necesidades subjetivas y emocionales, independientemente de la verdad. Ese egocentrismo se manifiesta en varios niveles: intelectual, social, moral, lingüístico, etc. El pensamiento egocéntrico es involuntario y no es capaz de adaptarse a la realidad, asimila las acciones de acuerdo con su punto de vista personal. De ahí se entiende el desequilibrio entre asimilación y acomodación.

\section{Presentación y análisis de los resultados}

\section{Identificar los conflictos en las relaciones familiares}

En cuanto a la constitución de la familia, el $62 \%$ de los niños de 5 años y el $76 \%$ de 6 años de la escuela pública viven con sus padres, el $38 \%$ de los niños de 5 años y el 24\% de 6 años viven con uno de los padres o con familiares . En la escuela privada, el $92 \%$ de los niños de 5 años y el $94 \%$ de los niños de 6 años viven con sus padres, apenas el $8 \%$ de los niños de 5 años y el $6 \%$ de los niños de 6 años viven con uno de los padres o con parientes cercanos.

Cuando se preguntó a las familias de los niños de 5 años lo que ellos entendían por conflicto, el 50\% respondió que la divergencia de ideas, es decir, cuando cada individuo tiene su pensamiento o creencia acerca sobre determinado objeto o situación, discordando del pensamiento o creencia de otros. $30 \%$ dice que son palabras ofensivas, $20 \%$ lo identificaron como agresión física y $20 \%$ disputas personales. Las familias de los niños de 6 años mostraron la divergencia de ideas y los insultos personales como la mejor explicación para el conflicto, ambos con un 33\% cada uno. Las agresiones físicas y las disputas personales aparecieron enseguida junto con el $22 \%$.

Viviendo en sociedad, se puede ver que los conflictos son comunes en la vida conyugal. Sin embargo, cuando están bien comprendidos por sus pares, se convierten en fuente de madurez para la vida conyugal y pueden conducir a relaciones más estrechas entre sus miembros. Las divergencias de ideas discutidas con calma, con demostraciones de apoyo y de actitudes que favorezcan la resolución de problemas no generan reacciones negativas en los niños, por el contrario, los pueden llevar a entender que a través del diálogo se pueden superar las dificultades. Por otro lado, las luchas por el poder, las agresiones físicas $o$ verbales presenciadas pueden causar sufrimiento a los niños.

Con relación a los conflictos experimentados, el $60 \%$ de las familias de los niños de 5 años dijeron que los conflictos son frecuentes en sus hogares, mientras que el $40 \%$ dijo que no existían. En las familias de seis años, el $56 \%$ dijo que no existen, mientras que el $44 \%$ lo afirmó.

Los resultados de las investigaciones se direccionan para lo que Dessen; Polonia (2007) afirma ser de gran importancia, identificar los aspectos del funcionamiento del sistema familiar que pueden estar relacionados tanto con el buen funcionamiento emocional y cognitivo como a las psicopatologías. Los padres proporcionan, o deberían proporcionar, todo el apoyo necesario para que el desarrollo saludable ocurra, además de funcionar como mediadores de los asuntos sociales.

El motivo generador de conflictos presentados por las familias de los niños de 5 años más común, el $30 \%$ son causados por problemas 
financieros, el $10 \%$ de las diferencias en el pensamiento, el $10 \%$ por diferencias en los proyectos de vida y el $10 \%$ por traición. $40 \%$ de estas familias no respondieron a la pregunta. En las familias de seis años muestran un 33\% como palabras ofensivas, el $22 \%$ como discusiones y agresiones físicas y el $23 \%$ prefirió no contestar.

La calidad de los vínculos afectivos que se forman entre padres e hijos puede ser considerada como indicadora de un desarrollo sano y, en consecuencia, de interacciones y patrones de ajuste positivo en todos los ambientes en los que participan (Dessen, Polonia, 2007). Sin embargo, estos mismos lazos emocionales pueden obstaculizar el desarrollo del niño y causar problemas en su adaptación social.

La teoría de la disonancia cognitiva de Festinger (1957) puede justificar las contradicciones entre las cuestiones que rodean el tema relacionado con las ideas divergentes. Festinger (1957) afirma que "cogniciones contrarias sirven como estímulos para que la mente obtenga o produzca nuevos pensamientos o creencias, o modifique creencias preexistentes, con el fin de reducir la cantidad de disonancia (conflicto) entre las cogniciones". Cuando ocurre una disonancia, el individuo entra en conflicto mínimo y procura adoptar algunas maneras de salir de la incomodidad.

Sobre la gravedad de los conflictos presentados anteriormente, el $40 \%$ de las familias de cinco años dijo que eran graves, el 20\% lo calificaron como intrascendentes y el $40 \%$ dijeron que estaban dentro de límites normales. Las familias de los niños de 6 años respondieron que el 67\% de los conflictos vividos están dentro del rango normal, el 22\% de ellos son graves y apenas el 11\% dijeron que tenían poca importancia.

Si los problemas familiares (conflictos) no son bien manejados y la falta o escasez de relaciones saludables no están ajustadas, la carencia de papeles maternos y paternos puede debilitar la formación sana de las relaciones cognitivas y afectivas de los niños.

Piaget (1977, p.11) postula que "el pleno desarrollo de la personalidad sobre sus aspectos más intelectuales es indisociable del conjunto de relaciones afectivas, sociales y morales". A primera vista, la maduración de la personalidad parece depender, principalmente, de factores afectivos. Formar personalidades independientes moralmente solamente es posible a través de la educación. Piaget (1977, p.11) afirma que "adquirido el lenguaje, la socialización del pensamiento se manifiesta por el desarrollo y los conceptos y las relaciones y el establecimiento de normas". Así, el medio en el cual el individuo se inserta y la forma en que se desarrollará influirá en gran medida en su formación.

En la investigación aplicada, los conflictos verificados que fueron vivenciados por las familias de los niños de 5 años son causados por problemas financieros, diferencias en el pensamiento, diferencias en los proyectos de vida y por traición. Para las familias de seis años, se observó que las palabras ofensivas, discusiones y agresiones físicas se manifiestan habitualmente.

Benetti (2006) afirma que los conflictos entre la pareja pueden presentarse a través de discusiones y peleas a escondidas, que se manifiestan por el boicot y la indiferencia, entre otros. Teniendo en cuenta que toda relación implica cierto nivel de conflicto, es importante entender qué aspectos del desarrollo del niño se ven afectados por ello.

\section{Verificar si los conflictos en las relaciones familiares pueden poner en peligro las relaciones interpersonales de los niños en fase de alfabetización}

Con respecto al comportamiento en el aula: el $92 \%$ de los niños de 5 años y el $90 \%$ de 6 años, de escuelas públicas demostraron ser afectivos con sus colegas, el $8 \%$ de los niños de 5 años y el 10\% de los niños de 6 años mostraron cierta agresividad. En la escuela privada, el $88 \%$ de los niños de 5 años y el 100\% de los niños de 6 años también demostraron ser afectivos, apenas el $12 \%$ de los niños de 5 años demostraron cierta agresividad. 
En cuanto al afecto demostrado a los profesores, el $93 \%$ de los niños de 5 años y el $80 \%$ de 6 años de las escuelas públicas demostraron ser afectivos con los profesores, el $7 \%$ de los niños de 5 años y el $20 \%$ de 6 años mostró cierta agresividad. En la escuela privada, el $88 \%$ de los niños de 5 años y el 100\% de los niños de 6 años también demostraron ser afectivos, apenas el $12 \%$ de los niños menores de 5 años mostraron baja afectividad.

Teniendo en cuenta la dificultad de concentración presentada en clase, el $24 \%$ de los niños de 5 años y el $46 \%$ de 6 años de la escuela pública no mostraron dificultad para concentrarse, el 76\% de los niños de 5 años y el 54\% de 6 años mostraron dificultades de concentración. En la escuela privada, el $88 \%$ de los niños de 5 años y el $81 \%$ de los niños de 6 mostraron un rango normal, apenas el $12 \%$ de los niños de 5 años y el $19 \%$ de 6 años se mostraron dispersos en el aula.

Sobre las dificultades de aprendizaje observadas, el 19\% de los niños de 5 años y el $60 \%$ de seis años de escuelas públicas no mostraron dificultades aparentes, en el $81 \%$ de los niños de 5 años y el $40 \%$ de 6 años se observaron limitaciones. En la escuela privada, el $88 \%$ de los niños de 5 años y el $84 \%$ de los niños de 6 años no demostraron dificultades, apenas el $12 \%$ de los niños de 5 años y el 16\% de los niños de 6 años presentaron limitaciones.

En cuanto a la presencia de la familia en la vida escolar del niño(a), se observó que el 11\% de los niños de 5 años y el $47 \%$ de 6 años de escuela pública no suelen ir acompañados de la familia, el $89 \%$ de los niños de 5 años y $53 \%$ de 6 años son acompañados por los responsables. En la escuela privada se observó que solamente el $4 \%$ de los niños de 5 años y $6 \%$ de los niños de 6 años no son acompañados por los responsables en las actividades escolares, el $96 \%$ de los niños de 5 años y el 94\% de los niños de 6 años son acompañados por la familia.

En cuanto a la participación de la familia en la escuela, el $76 \%$ de las familias de los niños de 5 años y el $46 \%$ de las familias de niños de 6 años de las escuelas públicas no participan en actividades promovidas por la escuela, el $24 \%$ de las familias de los niños de 5 años y el $44 \%$ de familias de niños de 6 años son participativos. En la escuela privada, apenas el $6 \%$ de las familias de niños de 5 años y $12 \%$ de las familias de niños de 6 años no participan, el 94\% de las familias de niños de 5 años y el $88 \%$ de las familias de niños de 6 años participan en actividades promovidas por la escuela.

Con respecto a la ayuda recibida por parte de las familias para la realización de las tareas en casa, el $62 \%$ de los niños de 5 años y el $39 \%$ de 6 años de las escuelas públicas reciben la ayuda, el $38 \%$ de los niños de 5 años y el $61 \%$ de 6 años hacen las tareas sin ayuda. En la escuela privada, el $94 \%$ de los niños de 5 años y el 83\% de los niños de 6 años reciben ayuda, solamente el $6 \%$ de los niños de 5 años y el 17\% de los niños de 6 años no reciben ayuda.

Se preguntó a las familias de los niños de 5 años sobre la forma de resolución de conflictos presentados, el $30 \%$ no respondió, el $20 \%$ dijo que salir de casa es una solución, el 10\% utiliza la fuerza física y el $40 \%$ busca resolverlos a través del diálogo. Para las familias de los niños de 6 años, el diálogo aparece con un 55\% de opciones de respuesta y el $45 \%$ prefiere no contestar.

Los resultados del estudio corroboran la percepción de Piaget (1977) de que si hay afecto, existe la capacidad de desarrollar el respeto mutuo tan necesario y que a través de él, el aprendizaje fluye con más facilidad.

Los padres deberían ser los principales responsables por el apoyo emocional, formación moral y social de los niños, preparándolos para buscar el éxito en el aprendizaje escolar y en las relaciones que se construirán, guiándolos para hacer frente a las frustraciones y conflictos.

Las palabras tienen un gran significado, la comunicación efectiva disminuye la posibilidad de malentendidos. El diálogo es fundamental en la tarea de educar. Las relaciones que se rigen por el compromiso y el diálogo se fortalecen.

Esta observación está cerca de lo que Piaget (2006) clasificó como relaciones de coacción y 
relaciones de cooperación. El proceso de enseñanza y aprendizaje se asocia con las relaciones interpersonales. La familia, las relaciones sociales e institucionales están estrechamente relacionadas con los resultados finales de los avances o la inercia en los procesos de aprendizaje.

Entre las consecuencias de estos conflictos en el cambio de comportamientos de los niños de 5 años en el ambiente escolar, identificados por las familias, las más notables son el nerviosismo y la agresividad, ambos con un $30 \%$ cada uno, el $20 \%$ de los padres dijeron que no percibieron los hay cambios y el $20 \%$ prefirió no responder. Entre las familias de los niños de 6 años, el 34\% de ellas dijeron que sus hijos son agresivos, el $22 \%$ dijo que hay distracción y el $44 \%$ no percibe diferencias.

El niño pre-operacional necesita seguridad, estabilidad, afecto y comprensión para sentirse seguro frente a los procesos de aprendizaje. Un entorno desfavorable favorece la agresividad, sentimientos de incapacidad y, por tanto, un comportamiento antisocial.

La indiferencia de la familia frente a las carencias afectivas presentadas puede poner a los niños frente a problemas de relaciones y aprendizaje. La desorganización familiar, la falta de límites y afectos, la agresividad en las relaciones, las pérdidas, la falta de interacción con el medio y la pobreza son vistas como posibles causas de estos comportamientos. $\mathrm{Al}$ analizar las relaciones cada vez más distantes entre los miembros de la familia, vemos que los niños procuran la atención de los padres. En la sociedad actual, la situación escolar puede ser importante para que los padres constaten que sus hijos no están bien en sus actividades, lo que puede llamarles la atención hacia un cambio de enfoque en el entorno familiar.

La encuesta revela que los conflictos familiares pueden comprometer la relación interpersonal que es construida por los niños en la etapa de alfabetización. Al no entender claramente lo que sucede en la familia, el niño puede llegar a sentirse culpable por los problemas y demostrar intolerancia a las reglas que se presenten en relación con los profesores y compañeros.
Como corolario, corroborando a Piaget (1977), una autonomía intelectual no es posible sin una autonomía moral, pues ambas se sustentan en el respeto mutuo, el cual, a su vez, se basa en el respeto por uno mismo y en el reconocimiento del otro como sí mismo. Trabajar con este tema en una propuesta pedagógica presupone la preocupación por desarrollar acciones en las que escuchar, mirar, sentir y pulsar son fuerzas inmanentes que definen los niveles de las relaciones que se establecen entre los individuos, especialmente en acciones de cooperación.

\section{Analizar si los conflictos en las relaciones familiares interfieren en el aprendizaje de los niños}

Se preguntó a los padres de los niños de 5 años sobre cuáles son los gestos afectivos más practicados en sus hogares, el $34 \%$ respondió que el abrazo, el 21\% afirmó que la sonrisa facilita la convivencia, el $20 \%$ dijo que una mirada cariñosa, la atención disponible y la asistencia proporcionada en las tareas escolares demuestran cariño, el $10 \%$ afirmó que el contacto físico aproxima las relaciones, el $15 \%$ son indiferentes a la necesidad de demostrar afecto. Las familias de los niños de 6 años recalcaron el abrazo y la sonrisa con un $29 \%$ como las principales muestras de afecto practicadas, la atención y la disponibilidad para escuchar lo que el otro tiene que decir aparecieron en segunda opción con un $25 \%$, la mirada cariñosa y la ayuda prestada aparecieron con un $14 \%$ de preferencia, el contacto con $10 \%$ y el $22 \%$ de los encuestados dijeron que no tienen tiempo para ofrecer afecto a los niños.

El resultado de la investigación sugiere que parte de las familias analizadas sufre algún tipo de quebrantamiento. Esto causa preocupación, pues es en la familia donde se absorbe la base emocional que acompañará nuestras acciones para el resto de nuestras vidas y es con ella que aprendemos nociones de lo correcto e incorrecto, de ética y de ciudadanía. Dessen y Polonia (2007) considera al ser humano como un todo, siendo 
las emociones responsables del desarrollo de los seres humanos. A través del medio en el que el niño vive, sentimientos como alegría, tristeza, miedo, odio o amor, pasan a tener mayor o menor relevancia.

El desarrollo del niño se caracteriza por cambios de comportamientos que se consideran importantes para permitir que el niño aprenda nuevas habilidades, y pueden ser caracterizados como deseables o indeseables (Dessen, Polonia, 2007). Deseados o adecuados son los cambios que ocurren como consecuencia de la interacción del organismo con el ambiente, permitiendo al niño el contacto con situaciones importantes de aprendizaje que promueven el desarrollo. Los comportamientos no deseados se refieren a problemas de comportamiento resultantes del exceso o falta de situaciones que facilitan o amplían las posibilidades de aprendizaje, y por lo tanto, de desarrollo. Estos se manifiestan mediante la internalización o externalización de comportamientos (Benetti, 2006).

Como internalización de comportamientos, se destacan la retracción y la ansiedad, preocupación exagerada, tristeza, inseguridad, timidez, miedos, manifestaciones psicosomáticas y rechazo a la escuela, entre otros. Los comportamientos de externalización, sin embargo, están marcados por la impulsividad, la explosividad, agresividad, agitación, características desafiantes y antisociales como mentiras, robos, faltar a clases, falta de respeto, luchas y hostilidad en las relaciones (Benetti, 2006). Estos son comportamientos que dificultan la interacción del niño con el ambiente, generan conflictos y con frecuencia causan la ruptura de las interacciones. En general, en el contexto escolar, el comportamiento de externalización se identifica más fácilmente, probablemente por tener una mayor visibilidad e interferir con la dinámica de la clase.

En este sentido, la familia es esencial para asegurar la supervivencia y la protección integral de los hijos, independientemente de la estructura familiar o la forma en que se ha estructurado. Es en la familia que se propicia la construcción de lazos afectivos y la satisfacción de las necesidades individuales, es en ella que deben ser absorbidos los primeros saberes y profundizados los vínculos humanos.

Por lo tanto, es evidente que si el afecto no estuviera presente dentro de la familia, la motivación que impulsa al ser humano a la búsqueda del conocimiento puede verse comprometida. En la visión de Piaget (2006, p.16) el afecto juega un papel primordial en el funcionamiento de la inteligencia, "no se podría razonar sin experimentar ciertos sentimientos y, por otro lado, no existen afectos sin un mínimo de comprensión".

Es importante tener en cuenta que la familia y la escuela son entornos de desarrollo y aprendizaje humano que pueden actuar como propulsores o inhibidores. El estudio de las relaciones en cada contexto, y entre ellos, constituye una importante fuente de información, en la medida en la que permite identificar aspectos o condiciones que generan conflictos y ruidos en las comunicaciones y, en consecuencia, en los patrones de colaboración entre ellos. En este sentido, es importante tener en cuenta cómo la escuela y, específicamente, los profesores emplean las experiencias que los estudiantes tienen en casa.

Los lazos afectivos estructurados y consolidados, tanto en la escuela como en la familia, permiten que los individuos hagan frente a los conflictos, enfoques y situaciones derivadas de estos vínculos, aprendiendo a resolver problemas de manera conjunta o separada. En este proceso, las diferentes etapas de desarrollo, características de los miembros de la familia y también los diferentes segmentos de la escuela, son factores esenciales en la dirección de lograr cambios en las funciones de desarrollo de la persona, con un impacto directo en su experiencia académica y psicológica.

\section{Consideraciones finales}

Después de la presentación y análisis de los resultados, comparándolos con la literatura, se observó que el aprendizaje de los niños puede verse 
comprometido por los conflictos en las relaciones familiares, debido a que el aprendizaje y el rendimiento escolar dependen, en primer lugar, de la interacción familiar $y$, posteriormente, de la relación entre el profesor y el estudiante. La teoría de Piaget (1977) reconoce a la afectividad como motivación para la actividad cognitiva y enfatiza que la afectividad y la razón son términos que se complementan entre sí. Para Piaget (1977) la afectividad es la energía, lo que mueve la acción, mientras que la razón es lo que permite al sujeto identificar deseos, sentimientos variados y obtener éxito en las acciones. En la teoría de Vygotsky (1993) se tiene un enfoque holístico, donde no hay forma de separar los intereses emocionales de los aspectos intelectuales.

La investigación muestra que las relaciones familiares se componen de una mezcla de conflicto y afecto. Por otra parte, estos conflictos pueden poner en peligro las relaciones interpersonales construidas por los niños en la etapa de alfabetización. Este hecho se establece en las relaciones de coacción y cooperación, señalados por (Piaget, 2006).

Los conflictos en las relaciones familiares interfieren en el aprendizaje de los niños, como se destaca en el estudio. Si el afecto no está presente dentro de la familia, la motivación que impulsa al ser humano en la búsqueda del conocimiento puede verse comprometida. Desde el punto de vista de Piaget (2006) el afecto desempeña un papel fundamental en el funcionamiento de la inteligencia.

Por lo tanto, a partir de la consideración de otros trabajos, como Cavenaghi y Saltini (2014), Dessen y Polonia (1992) y Benetti (2006), es evidente que a partir de Piaget, uno de los grandes desafíos de la educación sería fomentar el desarrollo intelectual en armonía con el desarrollo afectivo y moral, para que el sujeto pueda conquistar poco a poco su autonomía intelectual, emotiva y moral, basado en leyes de reciprocidad construidas en sus interacciones con el entorno físico, social, histórico y cultural. Así, el afecto y la cognición deben ser vistos de manera articulada, también en el campo de la educación. Se entiende que la afectividad y la inteligencia son aspectos indisociables, íntimamente vinculados e influenciados por la socialización, por el "vivir con el otro".

Por lo tanto, se entiende que el desarrollo de la inteligencia es a través del intercambio continuo entre el niño y el medio, ya que son las nuevas experiencias la que permiten la construcción de nuevas estructuras cognitivas a partir de las anteriores, hacia una organización mental cada vez más amplia.

La encuesta reveló un distanciamiento de los padres en relación a la vida escolar de los hijos, así como la presencia positiva de la mayoría de los padres en la formación no se notó presente. Tratar del desarrollo humano es también tratar de las relaciones que se establecen, así como de su estructura familiar. A través del estudio, se encontró que, efectivamente, el afecto y las relaciones construidas a partir de ese ámbito, son imprescindibles para que el niño se desarrolle de forma segura y fiable, a fin de que pueda llegar a vivir de forma independiente. La base proporcionada por la familia, de ser segura, proporciona al niño los instrumentos facilitadores del pensamiento, $y$ por lo tanto, influye en la formación de un aprendizaje saludable. Incluso si las familias no tienen una buena situación económica, social y cultural, las relaciones emocionales y morales pueden ser construidas de forma que puedan ayudar a los niños en su proceso de maduración.

\section{Referencias bibliográficas}

Benetti, S. P. C. (2006). Conflito conjugal: impacto no desenvolvimento psicológico da criança e do adolescente. Psicologia: Reflexão e Crítica, 19(2), 261-268.

Brasil (1988). Constituição da República Federativa do Brasil. Disponível em <http://www.planalto. gov.br/ccivil_03/constituicao/constituicaocompilado.htm>. Acesso em 01/02/2016. 
(2015). Código Civil instituído pela Lei no 13105, de 16 de março de 2015. Disponível em < http://www.planalto.gov.br/ccivil_03/_ ato2015-2018/2015/lei/113105.htm>. Acesso em 19/01/2017.

Cavenaghi, D. B. \& Saltini, C. (2014). Relações entre a afetividade e a inteligência no desenvolvimento mental da criança. Rio de Janeiro: Wak Editora.

Dessen, M. A., \& Polonia, A. C. (2007). Família e escola. Paidéia, 17(36), 21-32.

Duarte, L. F. D. (1994). Horizontes do indivíduo e da ética no crepúsculo da família. In: Ribeiro, I. (Org.), Família e sociedade brasileira: desafios nos processos contemporâneos. Rio de Janeiro: Fundação João XXIII.

Festinger, L. (1957). Teoria da dissonância cognitiva de Leon Festinger. Disponível em <http:// ojs.c3sl.ufpr.br/ojs/index.php/educar/article/ view/36006>. Acesso em 01/02/2016.

Genofre, R. M. (1997). Família: uma história jurídica. In: Carvalho, M. do C. B. de (Org.), A família contemporânea em debate. 2. ed. São Paulo: EDUC.

Mioto, R. C. T. (1998). Família e saúde mental : contribuições para reflexão sobre processos familiares. Revista Katálysis, 2, 20-26, maio. Florianópolis,

Piaget, J. (1977). O julgamento Moral na criança. SP: Mestre Jou.

(2006). Seis estudos de psicologia. RJ: Forense.

Ribeiro, M. S. (1999). A questão da família na atualidade. Florianópolis: JOESC.

Richardson, R. J. (2010). Pesquisa Social: métodos e técnicas. 3 ed. São Paulo: Atlas.

Vygotsky, L.S. (1993). Pensamento e linguagem. São Paulo: Martins Fontes.

Yin, R. K. (2016). Pesquisa Qualitativa: do início ao fim. Porto Alegre: Penso.

Wallon, H. (1995). A evolução psicológica da criança. Lisboa, Edições 70.Illicachi, J. (2007). Discriminación discursiva y dominación étnica en la educación. Reporte de investigación. Quito, Ecuador: FLACSO. 